\title{
Photonic crystal fiber Bragg grating based sensors - opportunities for applications in healthcare
}

\author{
Francis Berghmans $^{*}$, Thomas Geernaert $^{\mathrm{a}}$, Sanne Sulejmani ${ }^{\mathrm{a}}$, Hugo Thienpont ${ }^{\mathrm{a}}$, \\ Geert Van Steenberge ${ }^{\mathrm{b}}$, Bram Van Hoe ${ }^{\mathrm{b}}$, Peter Dubruel ${ }^{\mathrm{c}}$, Waclaw Urbanczyk ${ }^{\mathrm{d}}$, Pawel Mergo ${ }^{\mathrm{e}}$, \\ David J. Webb ${ }^{\mathrm{f}}$, Kyriacos Kalli ${ }^{\mathrm{g}}$, Jan Van Roosbroeck ${ }^{\mathrm{h}}$, Kate Sugden ${ }^{\mathrm{f}, \mathrm{i}}$ \\ ${ }^{a}$ Vrije Universiteit Brussel, Pleinlaan 2, 1050 Brussels, Belgium; \\ ${ }^{\mathrm{b}}$ IMEC-Universiteit Gent-CMST, Technologiepark 914A, B-9052 Zwijnaarde, Belgium; \\ ${ }^{\mathrm{c}}$ Universiteit Gent, Krijgslaan 281, 9000 Gent, Belgium; \\ ${ }^{\mathrm{d}}$ Wroclaw University of Technology, Wybrzeze Wyspianskiego 27, 50-370 Wroclaw, Poland; \\ ${ }^{\mathrm{e}}$ Marie Curie-Sklodowska University, Pl. M. Curie-Sklodowskiej 3, 20-031 Lublin, Poland; \\ ${ }^{\mathrm{f}}$ Aston University, Aston Triangle, B4 7ET Birmingham, UK; \\ ${ }^{\mathrm{g}}$ Cyprus University of Technology, Archbishop Kyprianos 31, 50329 Lemesos, Cyprus; \\ ${ }^{\mathrm{h}}$ FBGS International, Bell Telephonelaan 2H, 2440 Geel, Belgium; \\ ${ }^{\mathrm{i}}$ Astasense Ltd., Torrin Drive 52, SY3 6AW Shrewsbury, UK
}

\begin{abstract}
We review the state-of-the-art in photonic crystal fiber (PCF) and microstructured polymer optical fiber (mPOF) based mechanical sensing. We first introduce how the unique properties of PCF can benefit Bragg grating based temperature insensitive pressure and transverse load sensing. Then we describe how the latest developments in mPOF Bragg grating technology can enhance optical fiber pressure sensing. Finally we explain how the integration of specialty fiber sensor technology with bio-compatible polymer based micro-technology provides great opportunities for fiber sensors in the field of healthcare.
\end{abstract}

Keywords: Photonic crystal fiber, microstructured optical fiber, optical fiber sensor, fiber Bragg grating, biocompatible, biomechanical, polymer, embedded, micro-technology

\section{INTRODUCTION}

The use of sensors in the processing industry, material manufacturing, civil engineering, transport and energy production becomes more and more important for collecting data on the influence of physical quantities. These quantities typically include temperature, pressure, strain or force. In many cases conventional electromechanical sensors, e.g. electrical strain gauges, are perfectly qualified for these tasks. Electrical sensors may nevertheless experience difficulties in performing properly under challenging circumstances, for example in the presence of sources of electromagnetic radiation such as high voltage lines or lightning strikes. Their intrinsic temperature sensitivity can also disturb the sensor signal when the physical quantity of interest is measured in transient temperature regimes. Finally, electromechanical sensors can exhibit low fatigue life or drift, which calls for frequent replacement or recalibrations. When traditional sensors fail optical fiber sensors (OFS) can provide a solution. An optical fiber sensor encodes a measurand of interest in one (or more) properties of an optical signal that is guided within an optical fiber ${ }^{1}$. OFS feature many advantages over conventional sensors: they are small and lightweight; they are chemically inert; they can be multiplexed and allow distributed and/or quasidistributed sensor configurations; they allow absolute measurements because they can be self-referencing; and they can have a very linear response. The implementation and commercial success of this sensor technology has therefore considerably increased in the last 25 years and it is now being adopted steadily in the medical field as well. Reasons for this include for example the minimally invasive nature of optical fibers which makes them very suited for integration with instrumented catheters and their insensitivity to electromagnetic interference which allows using them in the presence of high magnetic fields encountered for example during magnetic resonance imaging procedures ${ }^{2}$.

*fberghma@vub.ac.be; phone 322 6293453; fax 322 6293450; www.b-phot.org

Optical Sensors and Biophotonics III, edited by Jürgen Popp, Dennis L. Matthews, Jie Tian, Chih-Chung Yang,

Proc. of SPIE-OSA-IEEE Asia Communications and Photonics, Vol. 8311, 831102 - (c) 2011 SPIE-OSA-IEEE

CCC code: $0277-786 X / 11 / \$ 18 \cdot$ doi: $10.1117 / 12.901240$ 
Three technological trends in the field of optical fiber sensors are very much likely to push the adoption of OFS for medical applications. First, the use of photonic crystal fibers which allow for unprecedented design flexibility of waveguide and sensing features ${ }^{3,4}$. Second, the use of polymer optical fibers which can withstand much larger mechanical deformations before breaking than their silica counterparts and which are inherently more bio-compatible $\mathrm{e}^{5,6}$. Third, the embedding of OFS in flexible and stretchable polymer materials that provides solutions for wearable biomedical sensor devices ${ }^{7-9}$.

Our most recent results obtained with these three technological developments are discussed in the sections below. In section 2 we first describe the basic sensor elements that we have developed. These are fiber Bragg gratings (FBGs) in two types of specialty fibers: glass photonic crystal fibers (PCFs) and microstructured polymer optical fibers (mPOFs). Section 3 then explains how these fibers are embedded within polymer materials to form sensor devices adapted to different applications in the field of healthcare.

\section{FIBER BRAGG GRATINGS IN SPECIALTY FIBERS}

One of the most well-known possibilities to turn an optical fiber into a sensor is to use a so-called fiber Bragg grating $(\mathrm{FBG})^{10,11}$. This device is a wavelength-selective filter fabricated inside the core of an optical fiber for which the reflected wavelength changes under the influence of external perturbations. Although these sensors have many advantages they still show a number of shortcomings. For example, a FBG in a conventional optical fiber is sensitive to both temperature changes and axial strain leading to cross-sensitivity problems. Furthermore the sensitivity of FBGs in conventional optical fiber to transverse strain remains considerably lower than to axial strain, while monitoring transverse strains is most important in many applications. Finally the measurable strain range is limited by the elastic limit of silica fiber. We therefore first report possible solutions to these issues.

\subsection{Bragg grating sensors in glass photonic crystal fibers}

We designed and fabricated a dedicated highly birefringent photonic crystal fiber (PCF). A Bragg grating inscribed in this PCF yields two Bragg reflection peaks and encodes pressure or transverse mechanical load in the spectral distance between those peaks. The latter is practically insensitive to temperature and to longitudinal strain. This principle is illustrated in Fig. 1.
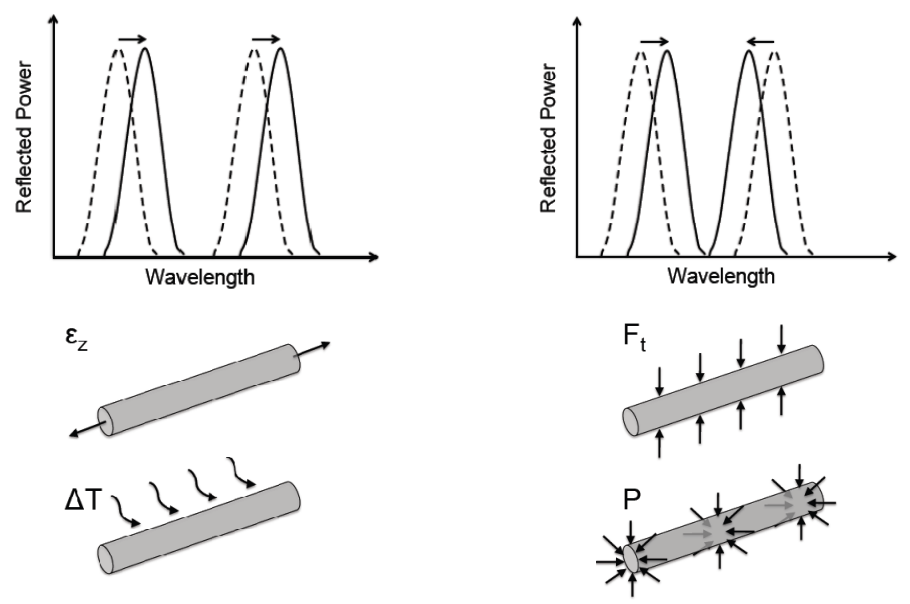

(a)

(b)

Figure 1. Illustration of the sensing principle exploited with FBGs in highly birefringent glass PCF. The measurand (pressure or a load applied transversally to the fiber) is encoded in the spacing $\Delta \lambda$ between the two Bragg peaks reflected by a FBG. (a) Response of the sensor to temperature changes or longitudinal strain as common mode signal - both peaks move in the same way and $\Delta \lambda$ remains unchanged. (b) Response of the sensor to transverse perturbations as differential signal - the peaks move in opposite direction and $\Delta \lambda$ changes.

The cross-section of the PCF ${ }^{12,13}$ designed for this purpose is shown in Fig. 2(a) and Fig. 2(b). We refer to this PCF as "Butterfly" PCF owing to the shape of the airhole microstructure. The central part of the core region is doped with $\mathrm{GeO}_{2}$ to allow the inscription of FBGs using conventional ultraviolet grating writing techniques. 


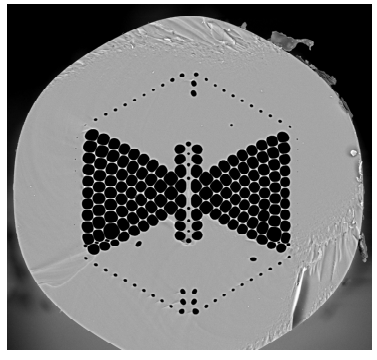

(a)

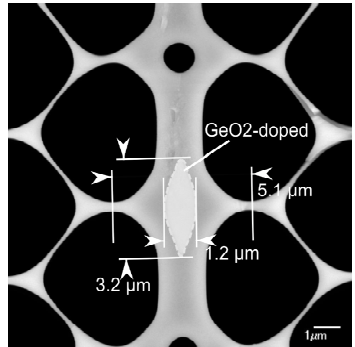

(b)

Figure 2. (a) Scanning Electron Microscope picture of the cross-section of the highly birefringent Butterfly PCF.

(b) Close-up of the core region of this PCF.

Fig. 3(a) shows reflection and transmission spectra of a FBG fabricated in the Butterfly $\mathrm{PCF}^{14,15}$ : the two reflection peaks are clearly visible and the grating strength is adequate for most sensing applications. Fig. 3(b) shows the change of the Bragg peak separation $\Delta \lambda$ as a function of applied hydrostatic pressure $\mathrm{p}$ for two different designs of the Butterfly PCF. Both negative $(\mathrm{d} \Delta \lambda / \mathrm{dp}<0$ - the peak separation decreases with pressure) and positive ( $d \Delta \lambda / \mathrm{dp}>0$ - the peak separation increases with pressure) sensitivities can be obtained by controlling the fabrication process and the resulting microstructure deformations and air filling factor in the core region. We obtained experimental values of $-15 \mathrm{pm} / \mathrm{MPa}$ and $33 \mathrm{pm} / \mathrm{MPa}$ for respective fibers, in very good agreement with the numerically simulated sensitivity values. Finite element simulations were carried out using commercially available COMSOL Multiphysics software and rely on analyzing the hydrostatic pressure sensitivity of the modal birefringence. To do so changes in the stress distribution and deformations of the microstructure are taken into account. The details of the different steps in the simulation process have been explained in Martynkien et al. ${ }^{13}$ In addition to the record high pressure sensitivities the sensitivity to temperature T expressed as $\mathrm{d} \Delta \lambda / \mathrm{dT}$ is below $0.02 \mathrm{pm} /{ }^{\circ} \mathrm{C}$.

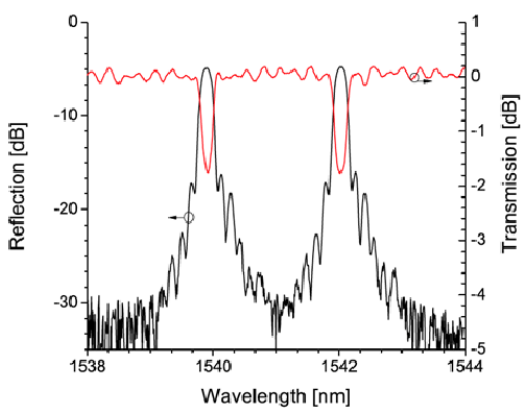

(a)

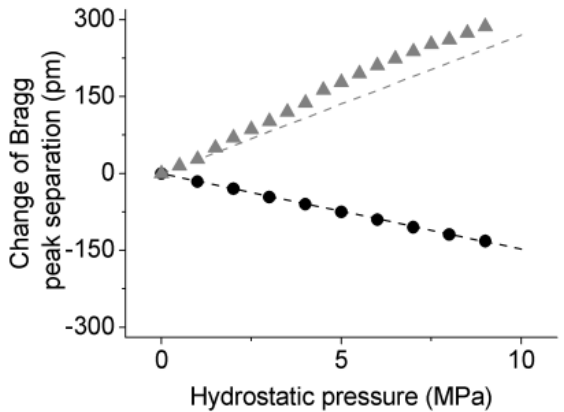

(b)

Figure 3. (a) Reflection and transmission spectra of a FBG fabricated in the $\mathrm{GeO}_{2}$-doped core of the Butterfly PCF from Fig. 2. (b) Bragg peak separation $\Delta \lambda$ versus hydrostatic pressure for two Butterfly PCFs with opposite pressure sensitivities. Triangles $(\mathrm{d} \Delta \lambda / \mathrm{dp}>0)$ and dots $(\mathrm{d} \Delta \lambda / \mathrm{dp}<0)$ are measurement values, while dotted lines are the corresponding numerical simulation results.

Record high sensitivities of this spectral distance to transverse strain up to $0.3 \mathrm{pm} / \mu$ strain when the sensors are embedded in carbon fiber reinforced composite material are within reach ${ }^{16,17}$. This sensitivity level also allows considering applications in the field of healthcare when such fibers are embedded in softer polymer materials (see section 3).

\subsection{Bragg grating sensors in polymer optical fibers}

The field of OFS can also greatly benefit from plastic optical fiber (POF) technology. Polymer OFS try to take advantage of plastic's ability to easily bind with organics, of its bio-compatibility in some cases and of the higher elastic limit and lower Young's modulus of the material compared to silica. Whilst POF had already been proposed for different sensor applications ${ }^{5}$, the use of POF Bragg grating (POFBG) sensors was until recently limited to laboratory demonstrations. There were two fundamental reasons behind this lack of development. First POFBGs were fabricated in the $1550 \mathrm{~nm}$ 
spectral region often exploited for telecommunications purposes ${ }^{18-20}$. This wavelength region was used because silica fiber has its lowest loss here. However it is a poor choice for POF because the attenuation of plastic fiber rises rapidly as the wavelength increases beyond the visible such that at $1550 \mathrm{~nm}$ the fiber loss is around $1 \mathrm{~dB} / \mathrm{cm}$. Such high losses limit the practical length of POF that can be used at this wavelength to typically less than $10 \mathrm{~cm}$. The second issue is that, regardless of the length of the POF that could be used, at some stage there needs to be a connection to silica fiber. This is because the fiber related technology that needs to be integrated with a POFBG to make it useful (pigtailed sources and detectors, couplers, circulators) all tends to be based on silica fiber. The fact that only a short length of POF can be used exacerbates this problem, requiring a silica connecting lead to be used unless the sensor is mounted right next to the measurement unit.

We solved these issues first by demonstrating the fabrication of POFBGs at $827 \mathrm{~nm}$ in a few-moded $50 \mu \mathrm{m}$ core microstructured POF using a HeCd laser and phase mask inscription set-up ${ }^{21}$. As a result of this development it became possible to record gratings in $25 \mathrm{~cm}$ lengths of fiber which is already much more convenient for practical applications. The reflection spectrum of a $827 \mathrm{~nm}$ grating in mPOF in shown in Fig. 4(a). Furthermore we succeeded in fabricating multiplexed sensors with up to 3 gratings for the very first time in the $850 \mathrm{~nm}$ spectral region, as shown in Fig. 4(b). The recording of multiple gratings can either be carried out using several phase masks or using an annealing approach. We found that to obtain stable POFBG behavior at temperatures above $55{ }^{\circ} \mathrm{C}$ the fiber had to be annealed, allowing shrinkage to take place. We realized that this process could be utilized to impart a well defined wavelength shift on a POFBG recorded in the fiber prior to annealing. This shift can either be used to create wavelength multiplexed POFBG sensors or it can be used to tune the wavelength of one sensor to accurately match a desired wavelength, for which no dedicated phase mask is available.

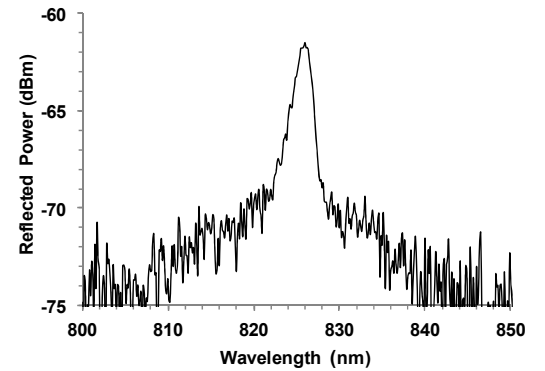

(a)

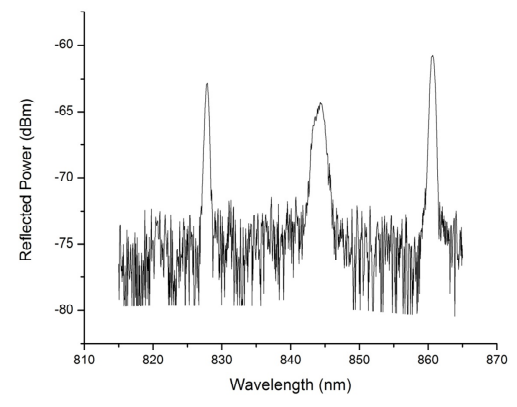

(b)

Figure 4. (a) Reflection spectrum of first POFBG in the low loss spectral region at $827 \mathrm{~nm}$. (b) 3 grating multiplexed sensor array in the $850 \mathrm{~nm}$ spectral region.

The connection issue was solved by using a UV curable glue to make a permanent connection between a silica fiber and POF. The precise procedure depends on the type of POF being used, but good success was achieved with both single mode step-index fiber and $50 \mu \mathrm{m}$ cored few-moded mPOF, where the gluing process typically adds rather less than $1 \mathrm{~dB}$ to the joint loss leading to an overall connection loss of about $4 \mathrm{~dB}$.

These important technological developments have enabled the POF gratings to be taken off the optical bench and into the field for the first time. Together with the high failure strain (typically larger than $50000 \mu$ strain) of such fibers this allows the consideration of different applications in healthcare as well (see section 3).

\section{EMBEDDED OPTICAL FIBER SENSORS}

Embedding the two types of sensors described above in polymer materials brings a realm of applications in the field of healthcare within range. One can for example embed FBGs in standard silica fibers in a 2D flexible bio-compatible polymer surface to form pressure sensing pads. Pressure on the pad is then transformed into axial strain detected by the FBG. The achieved operational and mechanical properties meet the requirements of various biomechanical applications targeting the measurement of pressure exerted on human skin, such as for example in amputee sockets, shoe sensors, wheelchair seating-system sensors, hospital-bed monitoring sensors, rehabilitation robots and wearable exo-skeletons ${ }^{22}$. 
The principle is illustrated in Fig. 5. Single or multiple FBGs can be embedded in polymer pads to form either small single pressure sensor elements or larger quasi-distributed pressure sensor surfaces.

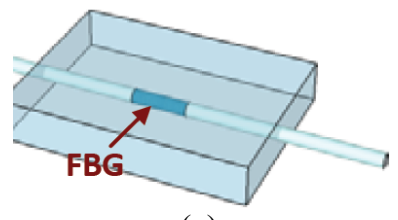

(a)

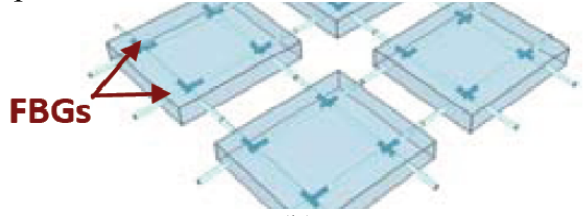

(b)

Figure 5. (a) Single FBG sensor element. (b) Multiple FBG pressure sensing surfaces.

To enable the embedding of fiber sensors in polymer pads we have developed different technologies that each come with their respective advantages ${ }^{8}$. They are illustrated in Fig. 6. The first embedding approach was to use straightforward injection molding in a polymethyl methacrylate (PMMA) or glass mold, during which the fibers are supported in channels located at the edge of the mold (Fig. 6(a)). The second approach was to use laser ablation to create tracks in a cured sheet of flexible host polymer. The main advantage of this approach is the flexibility in terms of fiber embedding design, which allows using designs from the field of micro-technology for stretchable (opto)electronics, incorporating meander shapes of non-stretchable materials (e.g. fibers) to allow for a certain level of elasticity (Fig. 6(b)). The third method is based on a soft lithography process for which the position of the optical fiber is defined even more accurately, by transferring a well defined negative master mold into the host material. For the master mold we used SU-8 (a hard epoxy material) patterning on a silicon wafer, since this allows for high accuracies in 3 dimensions, highly compatible with the requirements for fiber embedding. After patterning the SU-8 as part of the master mold, a controlled amount of Polydimethylsiloxane (PDMS) material is poured on the silicon wafer, which can be easily removed from the master mold after a moderate thermal curing step (Fig. 6(c)).

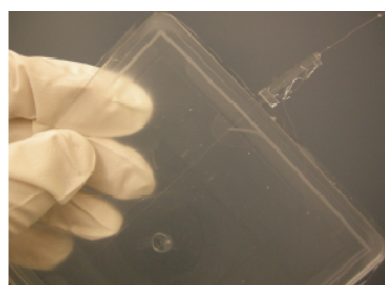

(a)

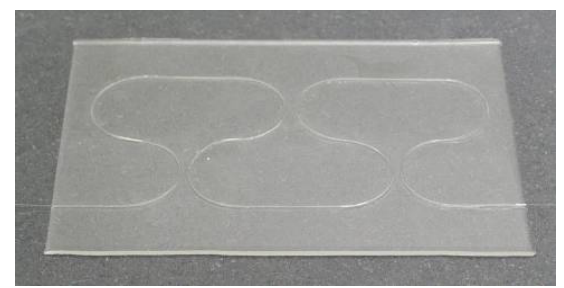

(b)

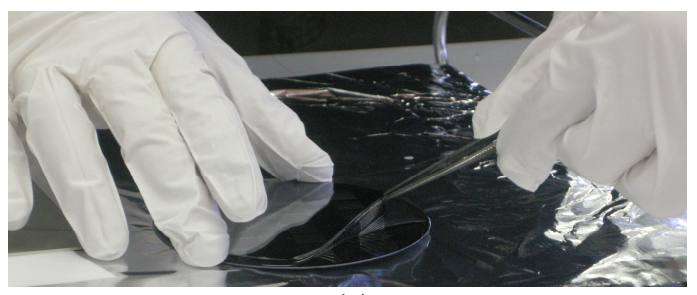

(c)

Figure 6. Fiber sensor pads fabricated using different techniques. (a) Molded glass fiber in thermally curable PDMS.

(b) PDMS laser ablated multiplexed sensor pad with a meandering fiber layout. (c) Soft lithography of PDMS material: peeling off of a structured PDMS layer.

Embedding technology is not limited to planar two-dimensional pads. The fiber sensors can also be embedded in other shapes of polymer materials, for example tubes as illustrated in Fig. 7. This again brings new possibilities to assemble sensor systems dedicated to medical applications such as gastro-intestinal pressure sensing (see section 3.2). Pressure applied on the tube is detected as strain acting on the fiber sensors in amounts that depend on the fiber location within the tube and on the diameter of the latter.

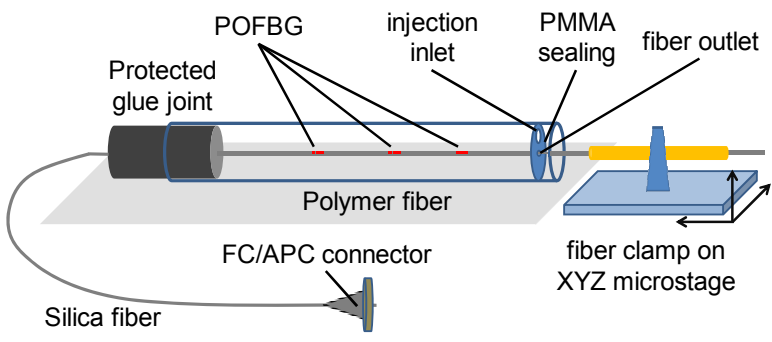

(a)

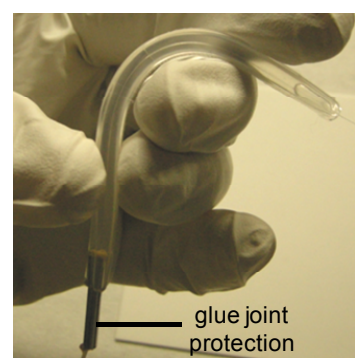

(b)

Figure 7. (a) Injection molding set-up for tubular embedding of fiber sensors. (b) Close-up of a polymer tube with embedded fiber. 


\subsection{Polymer embedded glass fiber sensors}

FBGs in highly birefringent PCFs as discussed in section 2.1 can be embedded in the polymer pads described above. Pressure or transverse strain is then directly detected by the sensor gratings which are insensitive to axial strain. This is illustrated in Fig. 8 that shows the results of two-dimensional finite element simulations carried out using commercially available COMSOL multiphysics software. One can calculate the material birefringence resulting in the Butterfly PCF from a transverse load applied to the polymer pad for different angular orientations of the PCF with respect to the direction of the applied load (Fig. 8(a)). This then leads to the calculation of the sensitivity of the Bragg peak separation to applied pressure as a function of the orientation, as shown in Fig. 8 (b). With this level of sensitivity, it is difficult to measure loads typically encountered in biomechanical applications which are on the order of tens of $\mathrm{kPa}$, as these lead to very small changes only in the Bragg peak separation. Although the Butterfly PCF has proven its importance for hydrostatic pressure sensing and transverse load sensing in its bare state, the results indicate that its high transverse load sensitivity is just not sufficient for most biomechanical applications when it is directly embedded in a flexible polymer pad. This is due first to the relatively small loads that are considered in this field and second, because the stress induced by the load is distributed over the entire pad. Hence the portion of stress actually transferred to the embedded fiber is limited.

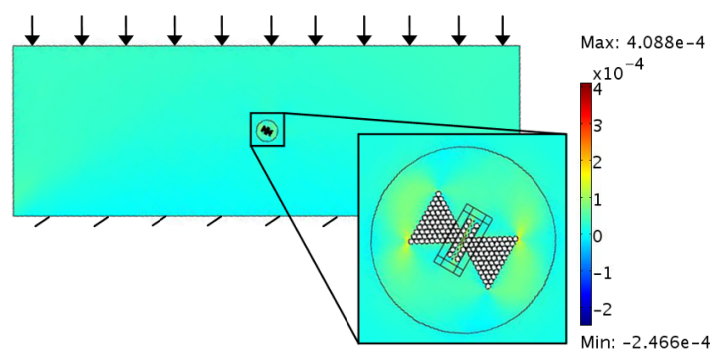

(a)

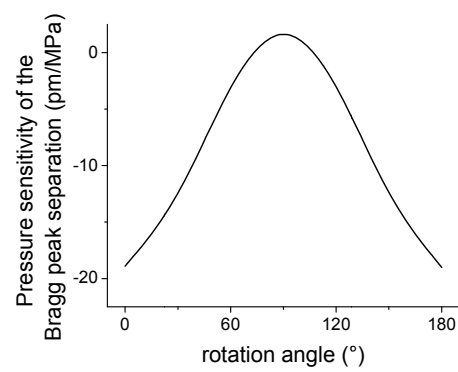

(b)

Figure 8. (a) Material birefringence in a Butterfly PCF oriented at $30^{\circ}$ due to a $1 \mathrm{MPa}$ transverse load applied to a polymer pad with elastic modulus $\mathrm{E}=615 \mathrm{kPa}$ and Poisson coefficient $v=0.49$.

(b) Pressure sensitivity $\mathrm{d} \Delta \lambda / \mathrm{dp}$ of an FBG in an embedded Butterfly PCF for different orientations.

Decreasing the mismatch in material properties between fiber and host material (e.g. use POF as discussed in section 3.2) is one possibility to increase the sensitivity of the pad. Another possibility is to structure the polymer pad in order to maximize the induced stress in the region around the optical fiber sensor. This is illustrated in Fig. 9 which shows the strain anisotropy that is present in a polymer pad with a PCF embedded in the centre when a distributed load is applied on the top surface while the bottom surface is fixed. Red regions are regions where the strain anisotropy is high, and thus regions that are favorable for positioning the fiber as its material birefringence will be most influenced there. Fig. 9(b) depicts the anisotropic strain when grooves are fabricated (e.g. with laser ablation) nearby the embedded fiber. Due to the presence of these macrostructures stress is concentrated resulting in an increased response to pressure.

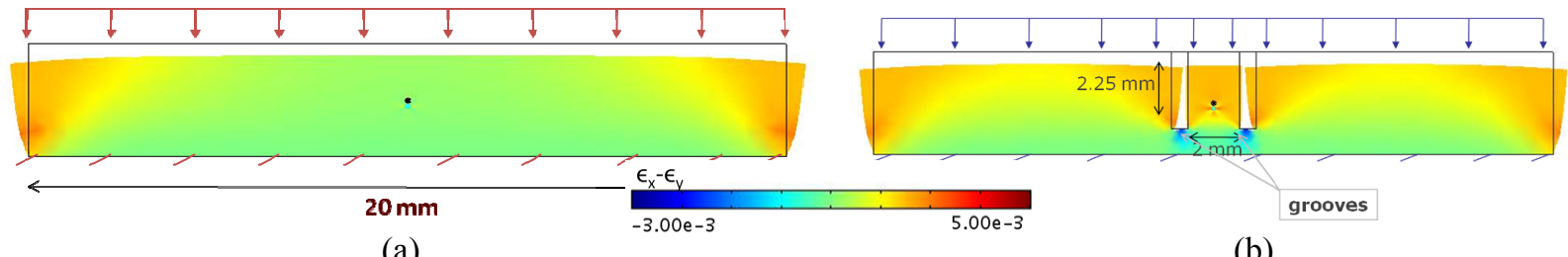

(a)

(b)

Figure 9. Strain difference $\varepsilon_{\mathrm{x}}-\varepsilon_{\mathrm{y}}$ present in a polymer pad with an embedded PCF.

(a) Plain structure. (b) Macrostructured with grooves.

Fig. 10 gives an overview of the possible improvement of the sensitivity of the Bragg peak separation of a Butterfly PCF when embedded in a polymer pad with various types of macrostructures. When no macrostructure is present (such as in Fig. 9(a)) the pressure sensitivity would be $-21 \mathrm{pm} / \mathrm{MPa}$. However by introducing a macrostructure (such as in Fig. 9(b)), 
this sensitivity can be increased with at least $73 \%$. This brings the response up to the level of biomechanical application requirements, i.e. from 35 to $50 \mathrm{pm} / \mathrm{MPa}$.

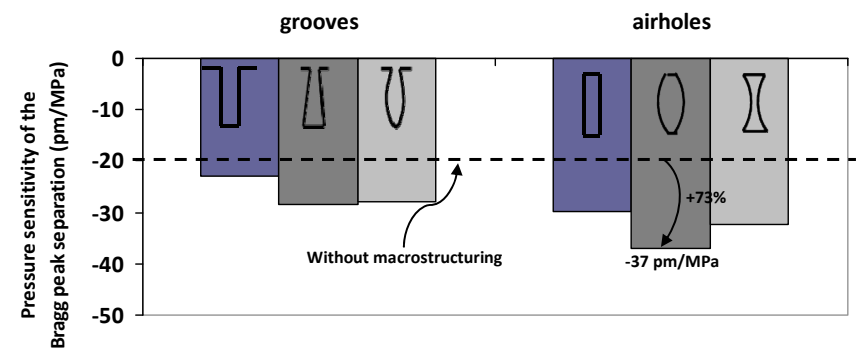

Figure 10. Overview of the possible improvement to the pressure response $d \Delta \lambda / \mathrm{dp}$ of embedded Butterfly PCF sensors when different types of macrostructures are applied.

\subsection{Polymer embedded polymer fiber sensors}

POFs with FBGs can also be embedded in polymer sensing pads made from a thermally curable PDMS transducer material (Sylgard 184® from Dow Corning). POFBGs prove to be more sensitive than regular glass FBGs (not to be confused with the FBGs in highly birefringent PCFs discussed in section 3.1) when embedded in a polymer pad. Table 1 below compares the response of POFBG and regular silica FBG to axial strain applied directly to the bare fiber and to the polymer pad containing the same fiber gratings. Although the response is of the same order of magnitude for bare fibers, the silica fiber behaves poorly even at modest strains in the millistrain range applied to the polymer pad. Slip and slide behavior may also lead to a considerable amount of hysteresis (Fig. 11(a)). The strain sensitivity is only about $0.03 \mathrm{pm} / \mu$ strain, which is a factor of 30 less than the sensitivity of the bare fiber. This is evidence that strain transfer from the elastic pad to the stiff silica fiber is poor. The POFBG exhibits a much more linear response with very little hysteresis and displays a sensitivity of $1.0 \mathrm{pm} / \mu$ strain when embedded (Fig. 11(b)). This is much closer to the bare fiber value of $1.5 \mathrm{pm} / \mu$ strain $^{23}$, but the difference remains significant and indicative of the disparity between the elastic moduli of POF and PDMS material. Therefore for mechanical strain sensing applications using polymer embedded FBGs, POFBGs can be considered a preferred solution.

Table 1. Comparison of the sensitivity to axial strain of a POFBG and a regular silica FBG

\begin{tabular}{|c|c|c|c|}
\hline \multicolumn{2}{|c|}{ Bare fiber sensitivity } & \multicolumn{2}{c|}{ Embedded fiber sensitivity } \\
\hline POFBG & Silica FBG & POFBG & Silica FBG \\
\hline $1.5 \mathrm{pm} / \mu$ strain $^{23}$ & $1.1 \mathrm{pm} / \mu$ strain $^{10}$ & $1.0 \mathrm{pm} / \mu$ strain & $0.03 \mathrm{pm} / \mu$ strain \\
\hline
\end{tabular}

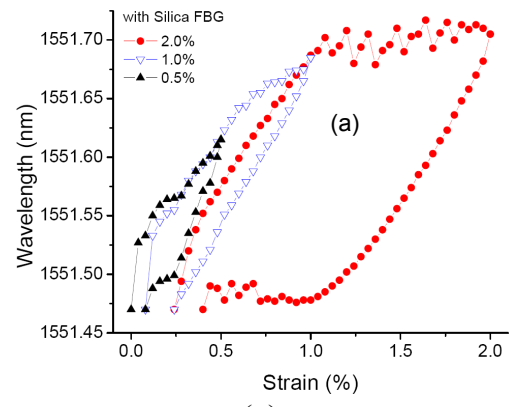

(a)

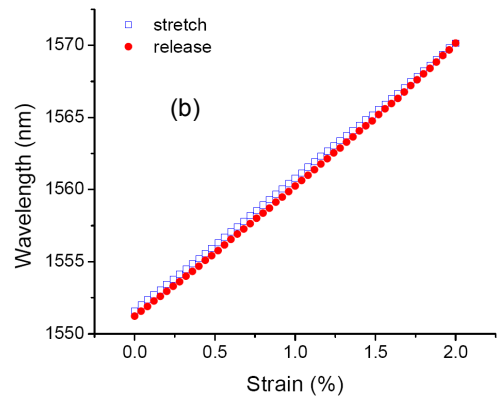

(b)

Figure 11. Bragg wavelength of an embedded FBG as a function of axial strain applied to the polymer pad.

(a) Silica FBG strained to $0.5,1.0$ and 2.0\%. (b) POFBG strained to $2 \%$.

Transverse load applied to the sensor pad will be translated into axial strain that is detected by the sensor grating. The response to contact pressure is assessed as illustrated in Fig. 12(a) with the pad lying on a hard flat surface, using a cylindrical post of mass $74 \mathrm{~g}$ and diameter $12 \mathrm{~mm}$ on which additional weights could be placed in various positions relative to the grating centered at position $[\mathrm{x}=0, \mathrm{y}=0]$. The lower elastic modulus of the POF results in a sensitivity 
approximately 10 times greater than the silica FBG (Fig. 12(b)). Fig. 12(c) indicates a linear relationship between the Bragg wavelength and the applied weight. The embedded POFBG response is therefore commensurate with biomechanical application requirements.

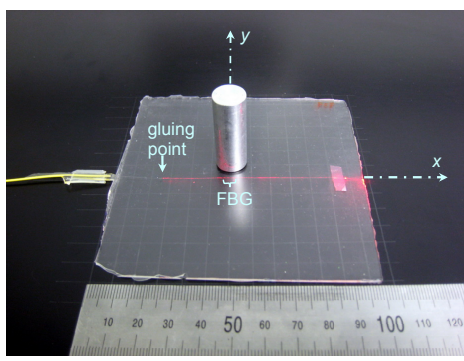

(a)

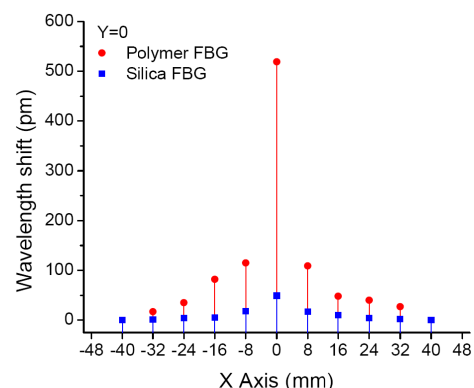

(b)

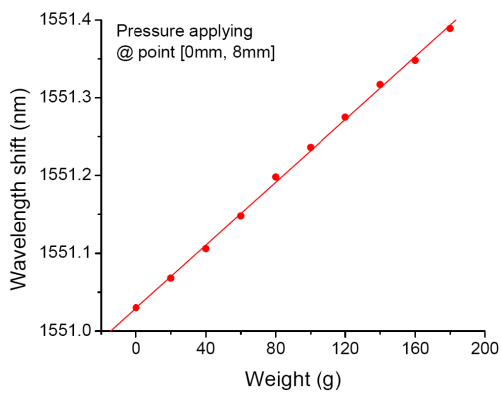

(c)

Figure 12. (a) Experimental arrangement for measuring the response to transverse load. (b) Response of embedded silica FBG and POFBG to pressure along the fiber axis. (c) Sensitivity to load of POFBG at a point laterally offset $8 \mathrm{~mm}$ from the grating position.

So far we discussed embedding in planar configurations only. Another possibility is to embed the sensors in tubes. Tubular embedding of a multiplexed array of POFBGs allows for example assembling an esophageal sensor system to measure pressure in a distributed manner along the esophagus when a patient swallows and to identify possible gastrointestinal disorders. As a proof-of-concept we have embedded an array of three wavelength multiplexed POFBGs in a PDMS tube and we have developed a low-cost optical interrogator system that allows the simultaneous read-out of the three sensor responses (Fig. 13(a)).

Fig. 13(b) shows the spectra of the multiplexed POFBGs before and after embedding and room temperature curing of the PDMS tube. This evidences that the POFBGs are still clearly visible after embedding. POFBGs also exhibit a more pronounced response than regular silica FBGs when embedded in the polymer tube. In terms of wavelength normalized sensitivity, the POFBG performs a factor of 5.7 better than a regular silica FBG as shown in Fig. 13(c). Owing to this enhanced response, the resolution requirements of the interrogation system for POFBGs are significantly relaxed compared to silica FBGs.

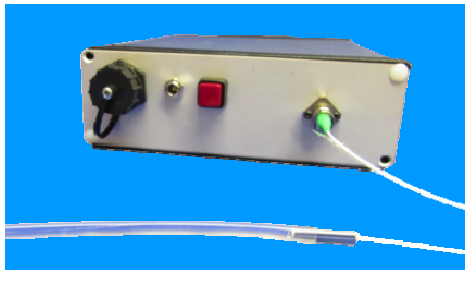

(a)

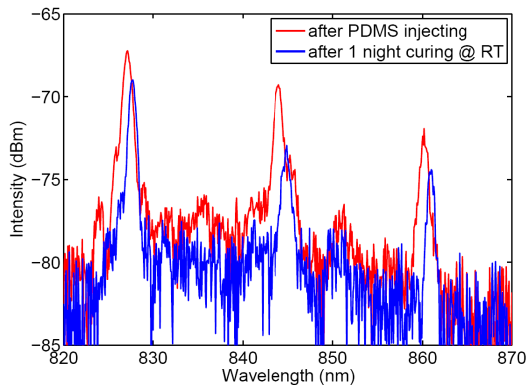

(b)

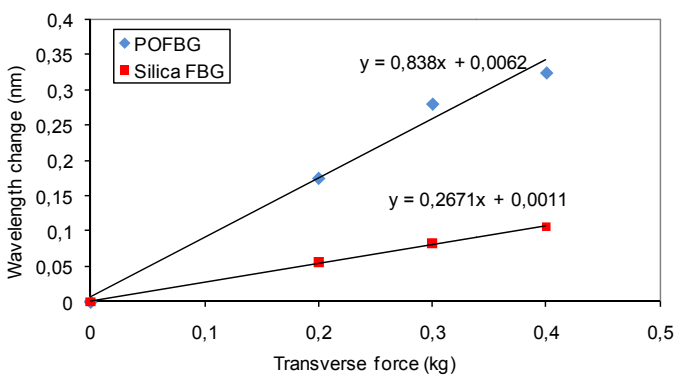

(b)

Figure 13. (a) Sensor read-out unit and tubular sensor array. (b) Spectra of the multiplexed POFBGs after embedding in and curing (at Room Temperature - RT) of the PDMS tube. (c) Bragg wavelength shift of tubular embedded POFBG (at $827 \mathrm{~nm}$ ) and silica FBG (at $1550 \mathrm{~nm}$ ) as a function of transverse load applied to the polymer tube.

The interrogation unit consists of a superluminescent diode emitting around $850 \mathrm{~nm}$ and a spectrometer integrated within a small table-top device shown in Fig. 13(a). The gratings shown are fabricated in $50 \mu \mathrm{m}$ core multimode mPOF but the system also works with singlemode devices. Using our interrogator the Bragg wavelength of the POFBGs can be resolved within $20 \mathrm{pm}$, leading to pressure measurement resolutions around $2.4 \mathrm{mmHg}$. For practical applications the required resolution is $1 \mathrm{mmHg}$, which could be achieved with our system should we use singlemode POF. 


\section{SUMMARY AND CONCLUSION}

We have introduced two specialty fiber technologies with great potential for application in the medical field. First we exploited the unprecedented design flexibility offered by photonic crystal glass fiber technology to design and fabricate Bragg grating based pressure sensor devices that operate in a temperature insensitive manner and that have a pressure sensitivity exceeding that of state-of-the-art bow-tie fiber based sensors with a factor of 20 . Second, we took advantage of the higher elastic limit and lower elastic modulus of polymer optical fibers compared with their silica counterparts. They can survive significantly higher strains and their low rigidity makes them ideal for monitoring flexible subjects. We fabricated fiber Bragg gratings in these polymer fibers in the $850 \mathrm{~nm}$ region where the fiber loss is 10 times lower than at $1550 \mathrm{~nm}$ and we developed a gluing technology to connect these polymer fibers to silica pigtails. By doing so we obtained POF sensor devices that can be used off the optical bench for the first time.

We then embedded these PCF and POF sensor devices in polymer pads to form sensor elements that can be applied in various biomechanical applications requiring distributed measurements of pressure exerted on the human skin with the intention to reliably address the interaction of the human body with external forces and hence to provide, for example, increased patient comfort or to avoid the development of pressure ulcers. We have developed different fiber embedding techniques based on molding, laser ablation or soft lithography to integrate multiplexed fiber sensors in stretchable polymer host materials. We proposed means to improve and to tune the sensitivity of the PCF sensor pads by structuring the polymer host and we have shown that POF sensor pads exhibit a large response to both axial strain and transverse load as compared to sensor pads using regular silica FBGs. Finally we have illustrated the potential of the POF technology by embedding a multiplexed array of POF Bragg gratings in a tube that provides a proof-of-concept demonstration for esophageal pressure sensing.

Whilst there is still progress to be made in the field of PCF and POF based sensors, we believe that these offer real advantages on different fronts. We are therefore confident that there is a future for these optical fiber sensor technologies in a wide range of medical applications. To ensure this future we will continue tackling essential issues such as packaging, fully-fledged system integration, optical coupling and interfacing, dependable strain transfer and reliability.

\section{REFERENCES}

[1] Berghmans, F. and Geernaert, T., "Optical Fibre Point Sensors", Advanced Fiber Optics: Concepts and Technology, Thévenaz, L., ed., EPFL Press, Lausanne, 308-344 (2011).

[2] Pinet, E., "Medical applications: saving lives", Nature Photonics 2(3), 150-152 (2008).

[3] Urbanczyk, W., Martynkien, T., Spulak, M., Statkiewicz, G., Olszewski, J., Golojuch, G., Wojcik, J., Mergo, P., Makara, M., Nasilowski, T., Berghmans, F., Thienpont, H, "Photonic crystal fibers: new opportunities for sensing", Proc. SPIE 6619, 66190G (2007).

[4] Frazao, O., Santos, J.L., Araujo, F.M., Ferreira, L.M., "Optical sensing with photonic crystal fibers”, Laser \& Photonics Review 2(6), 449-459 (2008).

[5] Kalli, K. and Webb, D.J., "Polymer Optical Fiber-Based Sensors", Advanced Fiber Optics: Concepts and Technology, Thévenaz, L., ed., EPFL Press, Lausanne, 345-388 (2011).

[6] Webb, D.J., "Polymer photonic crystal fibre for sensor applications", Proc. SPIE 7726, 77260Q (2010).

[7] Bosman, E., Missinne, J., Van Hoe, B., Van Steenberge, G., Kalathimekkad, S., Van Erps, J., Milenkov, I., Panajotov, K., Van Gijseghem, T., Dubruel, P., Thienpont, H., Van Daele, P., "Ultra thin optoelectronic device packaging in flexible carriers", IEEE Journal of Selected Topics in Quantum Electronics 17(3), 617-628 (2011).

[8] Van Hoe, B., Van Steenberge, G., Bosman, E., Missinne, J., Geernaert, T., Berghmans, F., Webb, D.J., Van Daele, P., "Optical fiber sensors embedded in flexible polymer foils", Proc. SPIE 7726, 772603 (2010).

[9] Chen, X., Zhang, C., Van Hoe, B., Webb, D.J., Kalli, K., Van Steenberge, G., Peng, G.-D., "Photonic Skin for Pressure and Strain Sensing", Proc. SPIE 7726, 772604 (2010).

[10] Othonos, A. and Kalli, K., [Fiber Bragg gratings: Fundamentals and Applications in Telecommunications and Sensing], Artech House, Boston \& London, 301-396 (1999).

[11] Cusano, A., Cutolo, A., Albert, J., [Fiber Bragg Grating Sensors: Recent Advancements, Industrial Applications and Market Exploitation], Bentham Science Publishers, Oak Park IL, 1-320 (2011). 
[12] Martynkien, T., Auszkiewicz, A., Statkiewicz, G., Olszewski, J., Golojuch, G., Szczurowski, M., Urbanczyk, W., Wojcik, J., Mergo, P., Makara, M., Nasilowski, T., Berghmans F., Thienpont, H., "Birefringent photonic crystal fibers with zero polarimetric sensitivity to temperature", Applied Physics B 94(4), 635-640 (2009).

[13] Martynkien, T., Statkiewicz-Barabach, G., Olszewski, J., Wojcik, J., Mergo, P., Geernaert, T., Sonnenfeld, C., Anuszkiewicz, A., Szczurowski, M.K., Tarnowski, K., Makara, M., Skorupski, K., Klimek, J., Poturaj, K., Urbanczyk, W., Nasilowski, T., Berghmans, F., Thienpont, H., "Highly birefringent microstructured fibers with enhanced sensitivity to hydrostatic pressure", Optics Express 18(14), 15113-15121 (2010).

[14] Geernaert, T., Nasilowski, T., Chah, K., Szpulak, M., Olszewski, J., Statkiewicz, G., Wojcik, J., Urbanczyk, W., Poturaj, K., Becker, M., Rothhardt, M., Bartelt, H., Berghmans F., Thienpont, H., "Fiber Bragg Gratings in Germanium-Doped Highly Birefringent Microstructured Optical Fibers", IEEE Photonics Technology Letters 20(8), 554-556 (2008).

[15] Geernaert, T., Becker, M., Nasilowski, T., Wojcik, J., Urbanczyk, W., Rothhardt, M., Bartelt, H., Terryn, H., Berghmans, F., Thienpont, H., "Bragg Grating Inscription in GeO2-Doped Microstructured Optical Fibers", IEEE Journal of Lightwave Technology 28(10), 1459-1467 (2010).

[16] Geernaert, T., Luyckx, G., Voet, E., Nasilowski, T., Chah, K., Becker, M., Bartelt, H., Urbanczyk, W., Wojcik, J., Degrieck, J., Terryn, H., Berghmans, F., Thienpont, H., "Transversal Load Sensing with Fiber Bragg Gratings in Microstructured Optical Fibers", IEEE Photonics Technology Letters 21(1), 6-8 (2009).

[17] Sonnenfeld, C., Sulejmani, S., Geernaert, T., Eve, S., Lammens, N., Luyckx, G., Voet, E., Degrieck, J., Urbanczyk, W., Mergo, P., Becker, M., Bartelt, H., Berghmans, F., Thienpont, H., "Microstructured Optical Fiber Sensors Embedded in a Laminate Composite for Smart Material Applications", Sensors 11(3), 2566-2579 (2011).

[18] Xiong, Z., Peng, G.-D., Wu, B., Chu, P.L., "Highly tunable Bragg gratings in single-mode polymer optical fibers", IEEE Photonics Technology Letter 11(3), 352-354 (1999).

[19] Dobb, H., Webb, D.J., Kalli, K., Argyros, A., Large, M.C.J., van Eijkelenborg, M.A, "Continuous wave ultraviolet light-induced fiber Bragg gratings in few- and single-mode microstructured polymer optical fibers", Optics Letters 30(24), 3296-3298 (2005).

[20] Chen, X., Zhang, C., Webb, D.J., Peng, G.-D., Kalli, K., "Bragg grating in polymer optical fibre for strain, bend and temperature sensing", Measurement Science and Technology 21(9), 94005 (2010).

[21] Johnson, I.P., Kalli, K., Webb, D.J., "827 nm Bragg grating sensor in multimode microstructured polymer optical fibre", Electronics Letters 46(17), 1217-1218 (2010).

[22] Kanellos, G.T., Papaioannou, G., Tsiokos, D., Mitrogiannis, C., Nianios, G., Pleros, N., "Two dimensional polymer-embedded quasi-distributed FBG pressure sensor for biomedical applications", Optics Express 18(1), 179-186 (2010).

[23] Peng, G.-D., Chu, P.L., "Polymer optical fiber photosensitivities and highly tunable fiber gratings", Fiber and Integrated Optics 19(4), 277-293 (2000).

\section{ACKNOWLEDGEMENTS}

This work was financially supported by the European Commission (EU) FP7 project "PHOSFOS" - Photonic Skins for Optical Sensing. Vrije Univ. Brussel also wishes to acknowledge financial support from the EU FP7 IAPP project "SMARTSOCKET", the Agency for Innovation by Science and Technology (IWT), the Research Foundation-Flanders (FWO), the Methusalem and Hercules Foundations Flanders, as well as the Interuniversity Attraction Poles (IAP)Belgian Science Policy. The COST TD1001 action "OFSESA" is acknowledged as well. 